\title{
Geometry effect on membrane absorption for CO2 capture. Part I: A hybrid modeling approach.
}

\author{
Kaiyun $\mathrm{Fu}^{1}$, Sunyang Wang ${ }^{1}$, Zhenbin $\mathrm{Gu}^{1}$, Yushu Liu ${ }^{1}$, Tianming Zai ${ }^{1}$, Shijie $\mathrm{Li}^{1}$, Xianfu \\ $\mathrm{Chen}^{1}$, Minghui Qiu ${ }^{2}$, and Yiqun Fan $^{1}$
}

${ }^{1}$ Nanjing Tech University

${ }^{2}$ Affiliation not available

November 10, 2020

\begin{abstract}
Membrane absorption (MA) has a great prospect for CO2 capture. In MA modeling, conventional 1D- and 2D- models make simplification of membrane contactor (MC) geometry. Geometry simplification allows an easy process modeling and numerical solution, however, is only reasonable for particular MCs. Here, efforts are underway to quantify the geometry effect on the MA-CO2 performance. First, we proposed a full 3D model without geometry simplification for simulating the MA-CO2 process in real MCs and then validated it with experimental data. More importantly, we highlighted a preferable hybrid model in which a correction factor $(\mathrm{F})$ was introduced to the $2 \mathrm{D}$ simulation results to make their combination approximately equal to the $3 \mathrm{D}$ simulation values. The $\mathrm{F}$ was correlated with dimensionless parameters obtained from computational fluid dynamics (CFD) studies for characterizing the geometry effect. Such hybrid modeling contributes to characterizing the influence of geometry on the MA-CO2 performance and improving computation accuracy-efficiency combinations.
\end{abstract}

\section{Hosted file}

Manuscript.pdf available at https://authorea.com/users/374665/articles/492108-geometryeffect-on-membrane-absorption-for-co2-capture-part-i-a-hybrid-modeling-approach 